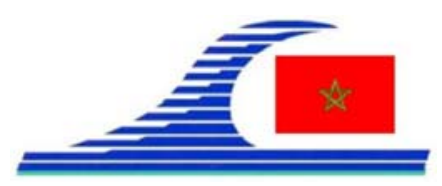

\author{
Conférence Méditerranéenne Côtière et Maritime \\ EDITION 2, TANGER, MAROC (2011)
}

Coastal and Maritime Mediterranean Conference

Disponible en ligne - http://www.paralia.fr - Available online

\title{
Evaluation de la bioaccumulation des métaux traces chez Donax trunculus dans la côte méditerranéenne marocaine (depuis Saidia à Al Hoceima)
}

\author{
Khalid EL BEKKAYE $^{1}$, Mohammed MELHAOUI ${ }^{1}$
}

1. Lab. d’hydrobiologie et écologie générale, Faculté des Sciences d’Oujda, Maroc.

Khalid_elbekkaye@hotmail.fr ; melhaouimohammed@yahoo.fr

\section{Résumé :}

Le suivi de la contamination côtière par les métaux, au moyen de bivalves mollusques filtreurs, est une pratique courante dans de nombreux programmes de surveillance de la pollution à travers le monde. Le présent travail vise à évaluer le degré de contamination de l'écosystème marin méditerranéen à travers une étude spatio-temporelle des teneurs en métaux traces chez Donax trunculus (Linné 1758) afin d'évaluer le risque sanitaire encouru par les prédateurs supérieurs, y compris l'être humain, consommateur des produits marins. La récolte de Donax trunculus a été effectuée au niveau de la Méditerranée marocaine, dans trois zones sableuses de faible profondeur, se trouvant entre les plages Saidia et Sfiha d'Al Hoceima. L'interprétation des résultats a montré l'impact de la pollution à l'eau de mer par les apports environnementaux sur les ressources halieutiques marines de la Méditerranée. Aussi l'analyse des variances montre des taux significatifs pour le fer et le plomb dans les trois stations, traduisant un rythme saisonnier de la bioaccumulation métallique. Ainsi, les concentrations de la majorité des métaux augmentent nettement en hiver.

Mots clés : Métaux lourds - Bioaccumulation - Donax trunculus - Méditerranée

\section{Introduction}

A travers des études récentes on constate une dégradation de plus en plus importante de la qualité de nos écosystèmes aquatiques, ce qui semble engendré par une pollution massive et anarchique, tant par les matières organiques naturelles que par les produits industriels de synthèse. Cette pollution semble affecter tous les compartiments de l'écosystème (BERG et al., 2009). Par cette étude on vise à quantifier la bioaccumulation de métaux traces chez un mollusque bivalve Donax trunculus (Linné 1758) de la côte méditerranéenne marocaine dans trois sites d'échantillonnage depuis plage Saidia jusqu’à plage Sfiha d'Al Hoceima. Les éléments détectés sont présent naturellement dans notre environnement, mais s'y rajoutent en permanence, en comparant les teneurs du substrat inerte à celles fixées dans un organisme vivant est justifié par leur capacité de fixer et d'accumuler de nombreux toxiques minéraux et organiques, ainsi constitue un outil très utile pour estimer le degré de pollution de l’hydrosystème (CASAS, 2004). 
La connaissance de la Mer :

un vecteur du développement durable en Méditerranée

\section{Présentation des zones d'étude}

Le Maroc représente un véritable carrefour entre l'Europe et l'Afrique, et entre la Méditerranée et l'Océan Atlantique, sa partie méditerranéenne appartenant à la mer d'Alboran (PROMAR, 2009).

- Station 1 : "Saidia" $\left(35,08939^{\circ} \mathrm{N} ; 02,23376^{\circ} \mathrm{W}\right)$ est une plage qui s'étend depuis l'Oued Kiss à la frontière Algéro-Marocaine jusqu'à la rive droite de l'estuaire de la Moulouya. C’est une plage sableuse de plus de $10 \mathrm{~km}$ ouverte et peu abritée.

- Station 2 : "Cap-de-l'eau" $\left(35,13704^{\circ} \mathrm{N} ; 02,40525 \mathrm{~W}^{\circ}\right)$ va depuis la rive gauche de l'embouchure de Oued Moulouya à la plage de Ras Kebdana. C'est une zone sableuse de 4 km abritée par les falaises d'Akemkoum el Baz, et le port de Cap-de-l'eau.

- Station 3 : "Al Hoceima" plage Sfiha $\left(35,21238^{\circ} \mathrm{N} ; 03,90222^{\circ} \mathrm{W}\right)$. C'est une zone sableuse et rocheuse sur plus de $2 \mathrm{~km}$, avec parfois des cailloutis.

\section{Matériels et méthodes}

L'échantillonnage a été réalisé à l'aide d'un râteau racleur dans les zones sableuses. Ce râteau se compose d'une armature métallique et d'une barre en métal sous forme de peigne supportant des dents de nombre et de longueur variables. Une poche en filet est attachée à l'armature. Elle a pour rôle d'accumuler et de stocker les captures (SHAFEE, 1999). Environ quarante individus adultes (taille moyenne environ $3 \mathrm{~cm}$ ), ont été récoltés et triés sur place, nettoyés puis stockés dans des flacons en polyéthylène, préalablement lavés, et contenant de l'eau du lieu de prélèvement. Les parties molles ont été lavées et égouttées. Ensuite elles ont été finement homogénéisées à l'aide d'un broyeur à couteau inox puis lyophilisées (BOUTHIR et al., 2004).

La prise de $0,5 \mathrm{~g}$ de poudre sèche de Donax trunculus a été minéralisée à $120^{\circ} \mathrm{C}$ pendant $4 \mathrm{~h}$ en présence de $4 \mathrm{~mL}$ d'acide nitrique (TAHIRI et al., 2005). L'analyse des échantillons a été réalisée par spectrophotométrie d'émission atomique (ICP-AES) suivant les méthodes AFNOR (1994) au laboratoire de l'UATRS de CNRST à Rabat.

\section{Résultats}

Dans notre étude, l'analyse des variances montre des coefficients significatifs pour tous les métaux, traduisant un rythme saisonnier de la bioaccumulation métallique. Ainsi, les concentrations de la majorité des métaux augmentent nettement en hiver et baissent en été, notamment pour le plomb alors que l'arsenic reste stable (figure 1). Quant aux variations spatiales observées dans la contamination métallique chez Donax trunculus, (LA VALLE et al., 2011) elles témoignent d'une contamination généralisée plus importante au niveau de la station d'Al Hoceima, particulièrement pour le fer (figure 2).

\section{Discussion}

Les mollusques filtreurs sont susceptibles de concentrer fortement les éléments métalliques à l'état de traces en milieu marin, cette bioaccumulation est due soit à un 
transfert direct par l'eau, soit à une désorption des éléments métalliques fixés sur les particules inertes ou vivantes en suspension dans l'eau qui leur servent de nourriture (CHAFI, 1995). Le degré de contamination le plus élevé durant les quatre saisons pour Donax trunculus correspond au plomb avec des taux supérieurs aux normes de la CEE (2001/2002), (Pb : 1,5 mg/kg), pendant l'hiver. Les mouvements des courants semblent aussi avoir une influence. Cependant, il se trouve que la côte méditerranéenne est soumise à de nombreux rejets permanents d'origine urbaine et industrielle, la proximité du port proche de Ras Kebdana, auxquels s'ajoutent ceux dûs à l'activité agricole pratiquée sur les rives de l'Oued Moulouya. Il est à signaler aussi que la majorité des unités industrielles qui cessent leurs activités pendant la saison estivale. Par ailleurs, le développement du secteur de la pêche, 1'installation d'industries et l'exode vers la frange côtière entraînent une surexploitation des ressources halieutiques. Cela va de pair avec une altération de la qualité de 1'environnement marin et la pollution ses écosystèmes. En particulier la consommation des bivalves contaminés soulève des problèmes toxicologiques pouvant porter préjudice à la santé publique (TAHIRI et al., 2005).

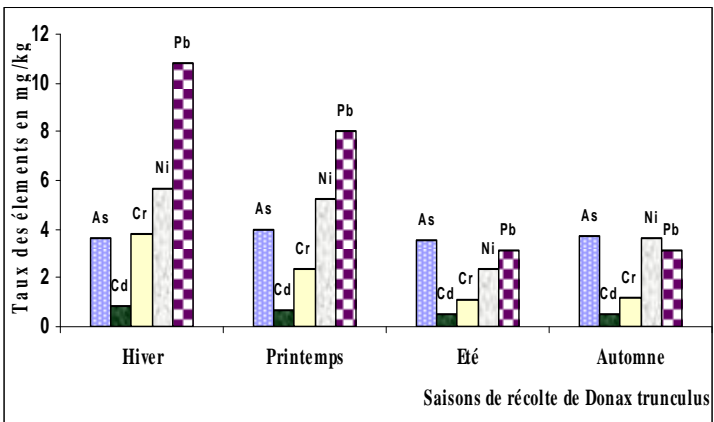

Figure 1. Teneurs des métaux (en $\mathrm{mg} / \mathrm{kg}$ ) durant les 4 saisons chez Donax au niveau de Cap-de-l'eau (Station 2).

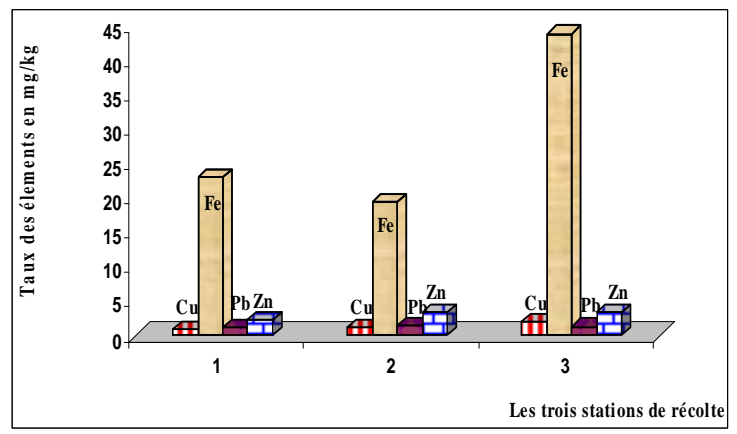

Figure 2. Teneurs des éléments (en $\mathrm{mg} / \mathrm{kg}$ ) chez Donax dans 3 stations différentes.

\section{Conclusion}

La croissance et le développement des bivalves dépendent de la disponibilité des nutriments qui fluctue saisonnièrement dans l'environnement et de l'intensité de leur stockage pour des raisons principalement physiologiques. C'est aussi le cas des métaux lourds. Le phénomène de bioaccumulation en particulier dans la glande digestive, (PELLERIN \& AMIARD, 2009) est maximal avant la reproduction et minimal après la ponte lorsque les réserves ont été épuisées au cours de la gamétogenèse. La période hivernale est souvent accompagnée d'un apport en éléments nutritifs qui pourraient conduire à une richesse en phytoplancton. Celui-ci est éventuellement contaminé par les métaux relargués par les sédiments et ceux issus des rejets industriels. 
La connaissance de la Mer :

un vecteur du développement durable en Méditerranée

Face à ce fléau s'impose la nécessité d'un contrôle régulier de la qualité de ces produits comestibles et d'une prise de mesure d'urgence pouvant aller jusqu'à l'interdiction formelle de la vente et de la consommation de ces produits prélevés dans l'estuaire de la Moulouya.

\section{Références bibliographiques}

AFNOR (1994). Essai physico-chimiques. Méthodes générales d'analyses et réactifs. T.2, 760 p.

BERG L.R., RAVEN P.H., HASSENZAHL D.M. (2009). Environnement. Editeur De Boek, 687 p.

BOUTHIR F.Z., CHAFIK A., BENBRAHIM S., SOUABI S., EL MERDHY H., MESSOUDI A., SIFEDDINE M. (2004). Qualité physico-chimique des eaux côtières du littoral de la wilaya du grand Casablanca (Océan Atlantique Marocain) utilisant la moule Mytilus galloprovincialis comme indicateur de la contamination métallique. Mar. Life - Maroc 2004, Vol. 14 (1-2), pp 59-70.

CASAS S. (2004). Modélisation de la bioaccumulation des métaux traces chez la moule, Mytilus galloprovincialis en milieu marin. Journées contamination décontamination des mollusques bivalves, Ifremer-Nantes.

CEE (2001/2002). Règlement de la commission (CE) $n^{\circ} 466 / 2001$, et $n^{\circ}$ 221/2002, portant fixation de teneurs maximales pour certains contaminants dans les denrées alimentaires. Journal officiel des communautés européennes.

CHAFI A. (1995). Mécanismes cellulaires de bioaccumulation des éléments minéraux toxiques chez certains organismes aquatiques de la méditerranée de l'oued Moulouya à l'oued Sebou du moyen Atlas : Toxicité de l'Aluminium et impact sur la santé humaine. FSO Maroc.

LA VALLE P., NICOLETTI L., FINOIA M.G., ARDIZZONE G.D. (2001). Donax trunculus (Bivalvia: Donacidae) as a potential biological indicator of grain-size variations in beach sediment. Ecological Indicators, pp 1426-1436.

PELLERIN J., AMIARD J.C. (2009). Comparaison of bioaccumulation of metals and induction of metallothioneins in two marine bivalves (Mytilus edulis and Mya arenaria). Comparative Biochemistry and Physiology; Part C: Toxicology \& Pharmacology, Volume 150, Issue 2, pp 186-195

PROMAR (2009). Biodiversité et échouements en mer d'Alboran, Almeria. Ecologistas en action, Rapport 2009.

SHAFEE M.S. (1999). Etude de la pêcherie des bivalves sur la côte méditerranéenne Marocaine. Institut Agronomie et Vétérinaire Hassan II Rabat.

TAHIRI L., BENNASSER L., EL ABIDI A., IDRISSI L., FEKHAOUI M., MORADI A. (2005). Contamination métallique de Mytilus galloprovincialis et des sédiments au niveau de l'estuaire de Bouregreg (Maroc). Water Qual. Res. J. Canada, Volume 40, $\mathrm{n}^{\circ} 1$, pp 111-119. 